\title{
Phase Noise and Performance Optimization in MUTC Photodetectors Using the Drift-Diffusion Equations
}

\author{
Seyed Ehsan Jamali Mahabadi, ${ }^{* 1}$ Franklyn J. Quinlan, ${ }^{2}$ Thomas F. Carruthers, ${ }^{1}$ and Curtis R. Menyuk ${ }^{1}$ \\ ${ }^{1}$ CSEE Department, University of Maryland, Baltimore County Baltimore, Maryland 21250, USA \\ ${ }^{2}$ National Institute of Standards and Technology, Boulder, Colorado 80305, USA \\ *sjamali1@umbc.edu
}

\begin{abstract}
We calculate the phase noise in modified unitraveling carrier (MUTC) photodetectors using the drift-diffusion equations, avoiding computationally-expensive Monte Carlo simulations. We optimize the performance over a range of device currents and biases.
\end{abstract}

Phase noise in photodetectors is a critical limiting factor in many RF-photonic applications [1], [2], particularly metrology applications in which phase noise limits the extent to which the low noise of an optical comb can be transferred to microwaves [2]. Quinlan et al. [3] predicted the phase noise is reduced when the photodetector is illuminated by short optical pulses, and experiments showed a significant reduction in the phase noise for short pulse detection [4]. However, the prediction in Ref. [3] that the phase noise tends to zero as the optical pulse tends to zero has not been realized, rather the decrease in phase noise ceases once the optical pulse becomes small compared to the duration of the electrical pulses that emerges from the photodetector. Sun et al. [5] were able to reproduce these experimental results using Monte Carlo simulations that accounted for collisional diffusion of electrons in the device. However, they did not take advantage of the fact that the distribution of electrons in any time slot is expected to be Poissonian, which simplifies the calculations and physical interpretation of the results. More practically, the Monte Carlo simulations are too computationally slow to be used for performance optimization.

Here, we use the drift-diffusion equations, combined with the observation that the arrival of electrons in any time interval is Poisson-distributed, to calculate the phase noise. This approach takes minutes on a desktop computer, as opposed to the many hours on a computer cluster that the Monte Carlo approach requires. We show that our results are consistent with earlier experiments [4] and Monte Carlo results [5]. We explain analytically that the mean-square phase noise tends to a constant non-zero value when the optical pulse duration tends to zero due to the shot-to-shot variation of the number of electrons in each time interval. Finally, we use our approach to study how the device parameters affect the phase noise and how to optimize the performance. We use a onedimensional (1-D) drift-diffusion model [6], [7] to calculate the impulse response of the modified unitraveling carrier (MUTC) photodetector; Fig. 1 shows its structure [8]. External loading, impact ionization, and the Franz-Keldysh effect are included in the model. Collision effects are included via the energy dependence of the drift and diffusion coefficients. Our starting point is the electron and hole continuity equations and the Poisson equation,

$$
\frac{\partial\left(p-N_{A}^{-}\right)}{\partial t}=-\frac{1}{q} \nabla \mathbf{J}_{p}+G_{\mathrm{ii}}+G_{\mathrm{opt}}-R(n, p),
$$

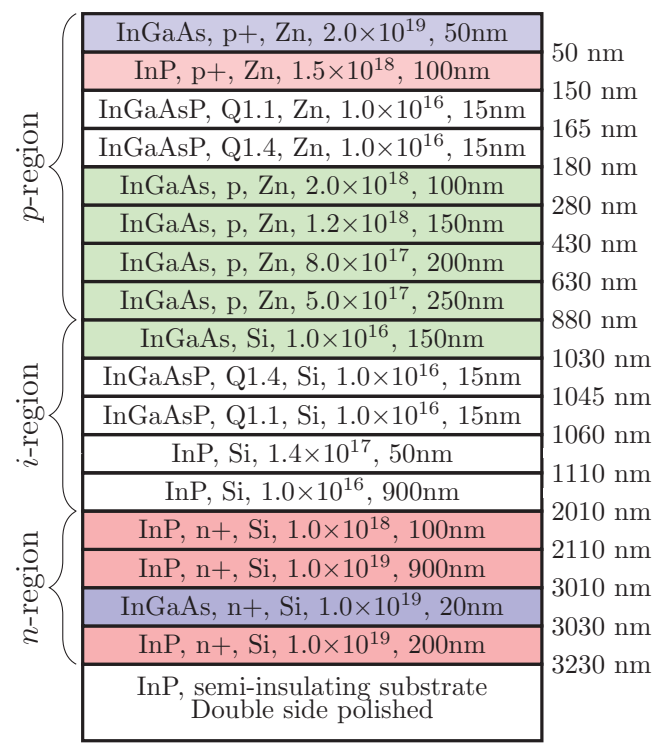

Fig. 1. Structure of the MUTC photodetector

$$
\begin{gathered}
\frac{\partial\left(n-N_{D}^{+}\right)}{\partial t}=+\frac{1}{q} \nabla \mathbf{J}_{n}+G_{\mathrm{ii}}+G_{\mathrm{opt}}-R(n, p), \\
\nabla \cdot \mathbf{E}=\frac{q}{\epsilon}\left(n-p+N_{A}^{-}-N_{D}^{+}\right),
\end{gathered}
$$

where $n$ is the electron density, $p$ is the hole density, $t$ is time, $q$ is the unit of charge, $\mathbf{J}_{n}$ is the electron current density, $\mathbf{J}_{p}$ is the hole current density, $R$ is the recombination rate, $G_{\mathrm{ii}}$ and $G_{\text {opt }}$ are impact ionization and optical generation rates, $\mathbf{E}$ is the electric field at any point in the device, $\epsilon$ is the permittivity of silicon, $N_{A}^{-}$is the ionized acceptor concentration, and $N_{D}^{+}$is the ionized donor concentration. The electron and hole current densities are governed by the equations

$$
\begin{aligned}
\mathbf{J}_{p} & =q p \mathbf{v}_{p}(\mathbf{E})-q D_{p} \nabla p, \\
\mathbf{J}_{n} & =q n \mathbf{v}_{n}(\mathbf{E})+q D_{n} \nabla n,
\end{aligned}
$$

where $\mathbf{v}_{n}(\mathbf{E})$ and $\mathbf{v}_{p}(\mathbf{E})$ are the electric-field-dependent electron and hole drift velocities, $D_{n}$ is the electron diffusion coefficient, and $D_{p}$ is the hole diffusion coefficient. The optical generation rate in Eq. 1 is

$$
G_{\text {opt }}=G_{c} \exp [-\alpha(L-x)],
$$

where $G_{c}$ and $\alpha$ are the generation rate coefficient and absorption coefficient, respectively, $x$ is distance across the device, and $L$ is the device length. 
We first calculate the steady-state parameters of the model: the hole density $p$, the electron density $n$, and the electrostatic potential $\psi$. We next perturb the generation rate by a small amount $\Delta G(t)$ over a time duration $t$ that is short compared to $h(t)$. We verified that the results are independent of our choice of $\Delta G(t)$ over a wide range of magnitudes and durations. Our calculated quantum efficiency $\eta=56 \%$, the relative intensity noise $(\mathrm{RIN})=-167 \mathrm{~dB} / \mathrm{Hz}$ for an average current $I_{\mathrm{avg}}=15$ $\mathrm{mA}$, and a 3-dB cut-off frequency of $25 \mathrm{GHz}$, are all in agreement with [5], [8].

We define $h(t)$ as the response of the photodetector to a finite-duration optical pulse, as in [4], [5]. Because the arrival of electrons in any interval $\Delta t$ varies from shot-to-shot and is Poisson-distributed, the number of electrons in that interval has a variance equals $h(t) N_{\text {tot }} \Delta t$, from which we calculate the phase noise. We find that when the optical pulse duration $\tau$ is less than about $500 \mathrm{fs}$ [9] $h(t)$ tends to a finite limit $h_{e}(t)$, which has a duration on the order of 10 ps. In this limit, we obtain

$$
\begin{gathered}
\left\langle\Phi_{m}^{2}\right\rangle=\frac{1}{N_{\text {tot }}} \frac{\int_{0}^{T_{R}} h(t) \sin ^{2}\left[2 \pi m\left(t-t_{c}\right) / T_{R}\right] \mathrm{d} t}{\left\{\int_{0}^{T_{R}} h(t) \cos \left[2 \pi m\left(t-t_{c}\right) / T_{R}\right] \mathrm{d} t\right\}^{2}} \\
\underset{h \rightarrow h_{e}}{\longrightarrow} \frac{1}{N_{\text {tot }}} \frac{\int_{0}^{T_{R}} h_{e}(t) \sin ^{2}\left[2 \pi m\left(t-t_{c}\right) / T_{R}\right] \mathrm{d} t}{\left\{\int_{0}^{T_{R}} h_{e}(t) \cos \left[2 \pi m\left(t-t_{c}\right) / T_{R}\right] \mathrm{d} t\right\}^{2}},
\end{gathered}
$$

where $\left\langle\Phi_{m}^{2}\right\rangle$ is the mean-square phase fluctuation, $N_{\text {tot }}$ is the total number of electrons in the photocurrent, $m$ is the harmonic number, and $t_{c}$ is the central time of the photocurrent. Hence, in the limit of short optical pulse durations, we find that $\left\langle\Phi_{m}^{2}\right\rangle$ tends to a non-zero constant.

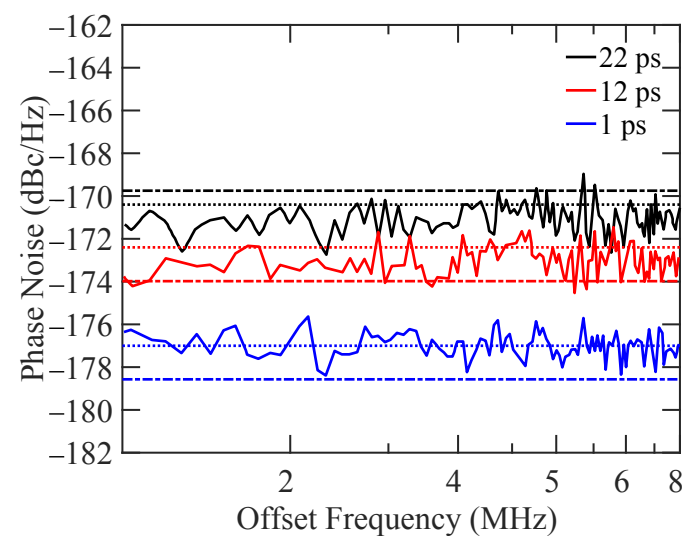

Fig. 2. Phase noise of the MUTC photodetector as a function of offset frequency for three different optical pulse widths. Dot-dashed lines are experimental results from [4]; solid lines are Monte Carlo simulation results from [5]; dotted lines are our simulation results.

Figure 2 shows the experimental and calculated phase noise of the MUTC photodetector as a function of offset frequency for three different optical pulse widths. Figure 3 shows the phase noise deviation from the long pulse limit as a function of pulse width. As shown in Figs. 2 and 3, we obtain good agreement with both experimental and Monte Carlo simulation results. Here, we considered a range of average currents between $14 \mathrm{~mA}$ and $18 \mathrm{~mA}$ and bias voltages between $15 \mathrm{~V}$

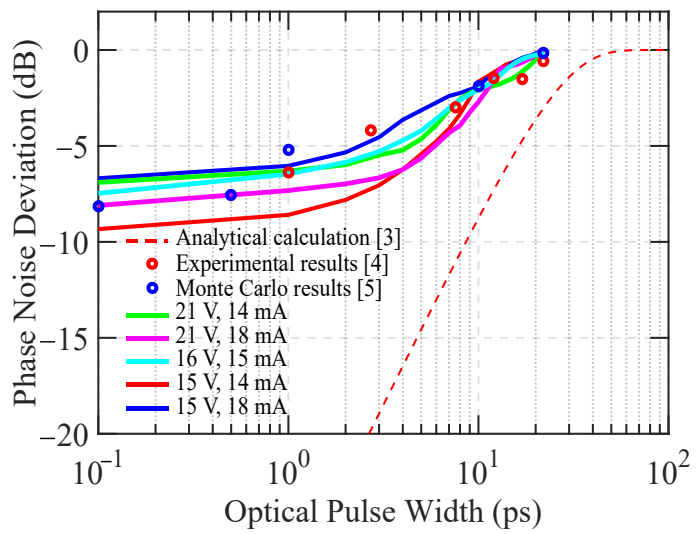

Fig. 3. Phase noise deviation from the long pulse limit.

and $21 \mathrm{~V}$, which corresponds to the range in the experiments of Quinlan et al. [4]. The lowest phase noise was achieved with an average current of $14 \mathrm{~mA}$ and a bias voltage of $15 \mathrm{~V}$.

In Fig. 3, we showed how the phase noise deviation from the long-pulse limit depends on the bias voltage and output current for the MUTC device considered earlier in [4], [5]. We have also explained physically why the phase noise approaches a constant value as the optical pulse duration approached zero. The computational rapidity of our approach allows us to carry out a detailed device optimization in which all the layer elements in the MUTC device are varied in order to minimize the phase noise while maintaining low nonlinearity. We will present the results of these studies.

\section{REFERENCES}

[1] Bahaa E. A. Saleh, Malvin Carl Teich, Fundamentals of Photonics (Wiley, New York, 1991).

[2] V. J. Urick, Keith J. Williams, and Jason D. McKinney, Fundamentals of Microwave Photonics (Wiley, New Jersey, 2015).

[3] F. Quinlan, T. M. Fortier, H. Jiang, and S. A. Diddams, "Analysis of shot noise in the detection of ultrashort optical pulses," J. Opt. Soc. Am. B 30, 1775-1785 (2013).

[4] F. Quinlan, T. M. Fortier, H. Jiang, A. Hati, C. Nelson, Y. Fu, J. C. Campbell, and S. A. Diddams, "Exploiting shot noise correlation in the photodetection of ultrashort optical pulse trains," Nat. Photonics 7, 290-293 (2013)

[5] W. Sun, F. Quinlan, T. M. Fortier, J. D. Deschenes, Y. Fu, Scott A. Diddams, and Joe C. Campbell, "Broadband noise limit in the photodetection of ultralow jitter optical pulses," Phys. Rev. Lett. 113, 203901 (2014).

[6] Y. Hu, C. R. Menyuk, X. Xie, M. N. Hutchinson, V. J. Urick, J. C. Campbell, and K. J. Williams, "Computational study of amplitude-tophase conversion in a modified unitraveling carrier photodetector,' IEEE Photon. J. 9, 5501111 (2017).

[7] Y. Hu, C. R. Menyuk, M. N. Hutchinson, V. J. Urick, and K. J. Williams, "Frequency dependent harmonic powers in a modified uni-traveling carrier photodetector," Opt. Lett. 42, 919-922 (2017).

[8] Z. Li, H. Pan, H. Chen, A. Beling, and J. C. Campbell, "High-saturationcurrent modified uni-traveling-carrier photodiode with cliff layer," IEEE J. Quantum Electron. 46, 626-632, (2010).

[9] Y. Hu, C. R. Menyuk, X. Xie, M. N. Hutchinson, V. J. Urick, J. C. Campbell, and K. J. Williams, "Computational study of amplitudeto-phase conversion in a Modified Uni-Traveling Carrier (MUTC) photodetector," IEEE Photon. J. 9, 5501111 (2017). 